\title{
Room Temperature Electrically Detected Nuclear Spin Coherence of NV Centres in Diamond
}

\author{
H. Morishita ${ }^{1,2^{*}}$, S. Kobayashi ${ }^{1,2}$, M. Fujiwara ${ }^{1,2}$, H. Kato ${ }^{2,3}$, T. Makino ${ }^{2,3}$, S. Yamasaki ${ }^{2,3}$ \& \\ N. Mizuochi ${ }^{1,2 *}$
}

We demonstrate electrical detection of the ${ }^{14} \mathrm{~N}$ nuclear spin coherence of $\mathrm{NV}$ centres at room temperature. Nuclear spins are candidates for quantum memories in quantum-information devices and quantum sensors, and hence the electrical detection of nuclear spin coherence is essential to develop and integrate such quantum devices. In the present study, we used a pulsed electrically detected electron-nuclear double resonance technique to measure the Rabi oscillations and coherence time $\left(T_{2}\right)$ of ${ }^{14} \mathrm{~N}$ nuclear spins in $\mathrm{NV}$ centres at room temperature. We observed $T_{2} \approx 0.9 \mathrm{~ms}$ at room temperature, however, this result should be taken as a lower limit due to limitations in the longitudinal relaxation time of the NV electron spins. Our results will pave the way for the development of novel electron- and nuclear-spin-based diamond quantum devices.

Nuclear spins in a semiconductor have a long coherence time $\left(T_{2}\right)$ due to the good isolation from environmental noise ${ }^{1-7}$. Therefore, they are candidates for quantum memories in quantum-information devices and quantum sensors. Using nuclear spins (e.g., nitrogen and carbon) in diamond for quantum memories, highly sensitive magnetic sensors ${ }^{8-10}$, quantum repeaters ${ }^{11}$, quantum registers ${ }^{12,13}$, etc., have been demonstrated at room temperature. In these demonstrations, the detection of nuclear spin coherence is essential. Nuclear spin coherence can be detected via the electron spins of nitrogen-vacancy (NV) centres, which also have a long $T_{2}$ at room temperature ${ }^{14-16}$. NV electron spins can be detected by optical techniques ${ }^{17}$ and electrical techniques ${ }^{18-20}$. The electrical technique is an important technology for developing and integrating quantum devices. Furthermore, a theoretical model predicts that its detection sensitivity is approximately three times higher than that of the optical technique $^{19}$. While the photoelectrical detection of the electron spin coherence of an ensemble of NV centres ${ }^{19,20}$ and photoelectrical coherent spin-state readout of single NV centres ${ }^{21}$ at room temperature has been demonstrated, the direct electrical detection of nuclear spin coherence remains challenging. This is because nuclear spins are well isolated from the environment and the interactions between nuclear spins and current are very weak. Thus, we focus on an electrically detected electron-nuclear double resonance (EDENDOR) technique to demonstrate room-temperature electrical detection of nuclear spin coherence.

The first EDENDOR measurement was demonstrated by Stich and collaborators for phosphorus (P) donors in silicon at $4.2 \mathrm{~K}^{22}$. After this demonstration, two other groups independently demonstrated pulse EDENDOR measurements of P-donors in silicon ${ }^{23-26}$. They measured Rabi oscillations and $T_{2}$ of $\mathrm{P}$-donor nuclear spins from 3.5 to $5 \mathrm{~K}$. Furthermore, pulsed EDENDOR measurements of proton nuclear spins in organic semiconductors have been demonstrated ${ }^{27}$. Proton nuclear spin resonances have been measured at room temperature, but Rabi and $T_{2}$ measurements of proton nuclear spins have not been reported yet. To the best of our knowledge, there have been no demonstrations of room-temperature electrical detection of nuclear spin coherence in diamond or any other materials.

The EDENDOR signals of ${ }^{14} \mathrm{~N}$ nuclear-spin coherence are observed by measuring the change in the electrically detected electron-spin echo (ESE) intensity of the NV centres. Here, the technique of electrical detection of magnetic resonance signals is called electrically detected magnetic resonance (EDMR). The EDMR of the NV centres measures the photocurrent change due to electron-spin resonances of the NV centres ${ }^{18-21}$. The photocurrent can be generated under illumination by a 532-nm laser via a two-photon ionisation process, depicted in Fig. 1(a). The

${ }^{1}$ Institute for Chemical Research, Kyoto University, Gokasho, Uji, Kyoto, 611-0011, Japan. ${ }^{2}$ CREST, Japan Science and Technology Agency, Kawaguchi, Saitama, 332-0012, Japan. ${ }^{3}$ Energy Technology Research Institute, National Institute of Advanced Industrial Science and Technology (AIST), Tsukuba, Ibaraki, 305-8568, Japan. *email: h-mori@ scl.kyoto-u.ac.jp; mizuochi@scl.kyoto-u.ac.jp 

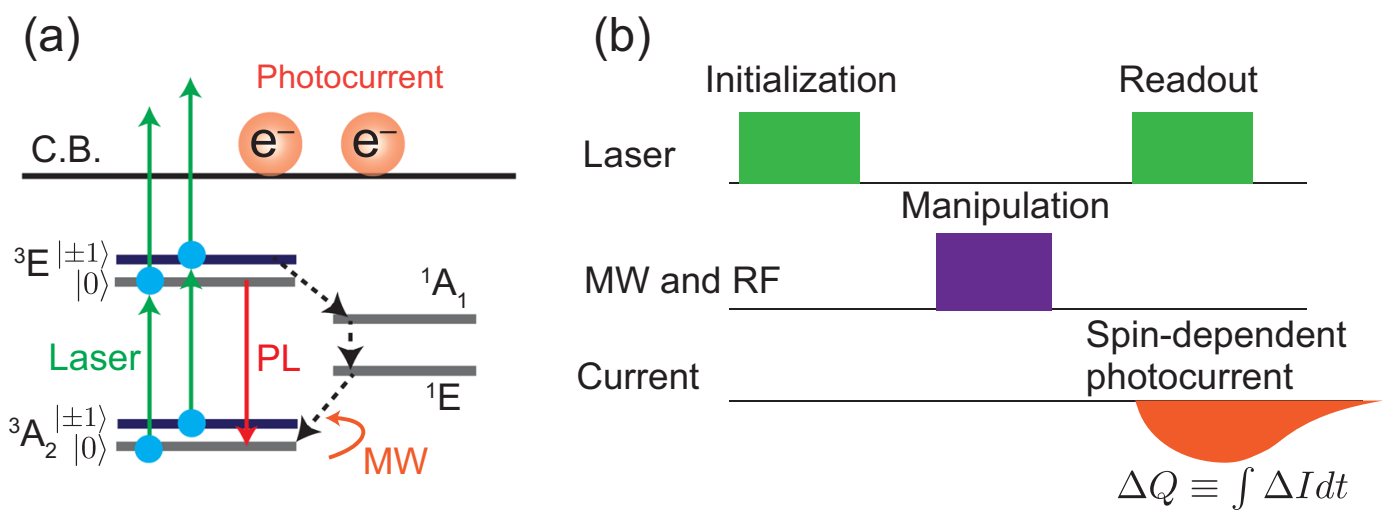

Figure 1. (a) Schematic of the EDENDOR measurements of NV centres in diamond. (b) Process for the EDENDOR measurements.
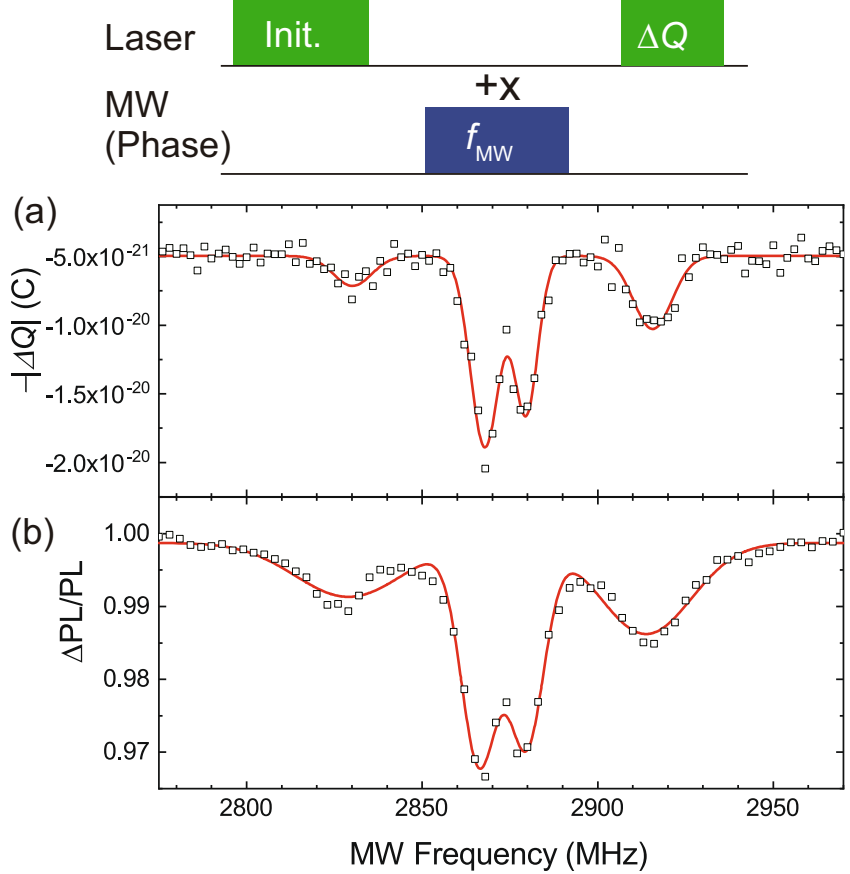

Figure 2. (Top) Pulse sequence for pEDMR and pODMR measurements. $+x$ indicates as the phase of MW pulse. (a) pEDMR spectrum. (b) pODMR spectrum.

figure shows that the $| \pm 1\rangle \mathrm{NV}$ electron spin at the ${ }^{3} E$ state has a transition probability to the long-lived ( $\left.220 \mathrm{~ns}\right)$ metastable ${ }^{1} E$ states, where $\left|m_{s}\right\rangle$ describes an NV electron spin. This causes the photocurrent to decrease due to a magnetic resonance transition from $|0\rangle$ to $| \pm 1\rangle$ after the optical initialisation to $|0\rangle^{18}$. Based on this mechanism, this study measures the electron and nuclear spin coherences with the pulse sequence depicted in Fig. 1(b). The details of the pulse sequence are as follows. (1) A 532-nm laser is used to initialise the NV electron spins to $|0\rangle$. (2) The electron and/or nuclear spins are manipulated by microwave (MW) and/or radiofrequency (RF), respectively. (3) The final laser is used to generate the photocurrent via two-photon ionisation, which contains the EDMR and EDENDOR signals. They are observed as a transient response, which is depicted in Fig. 1(b). Hence, this study defines the EDMR and EDENDOR intensities $(\Delta Q)$ as the change in the photocurrent $(\Delta I)$ integrated by the time during which the photocurrent is changing, which is described as $\Delta Q \equiv \int \Delta I d t^{27,28}$. Thus, the unit of the intensities of EDMR and EDENDOR can be described by coulomb $(\mathrm{C})^{27,28}$.

\section{Result}

Pulsed EDMR spectrum of ensemble of NV centre. Initially, we performed a pulsed EDMR (pEDMR) measurement with the pulse sequence depicted in the top of Fig. 2. We used $30 \mathrm{~mW}$ of laser power and an input MW power of $10 \mathrm{~mW}$ at a static magnetic field of $\sim 10 \mathrm{G}$ approximately along the [111] direction of the diamond crystal. Figure 2(a) shows the EDMR intensity $(\Delta Q)$ as a function of MW frequency, showing that four signals appeared. To analyse the observed spectrum, we measured the pulsed ODMR (pODMR) spectrum shown in 


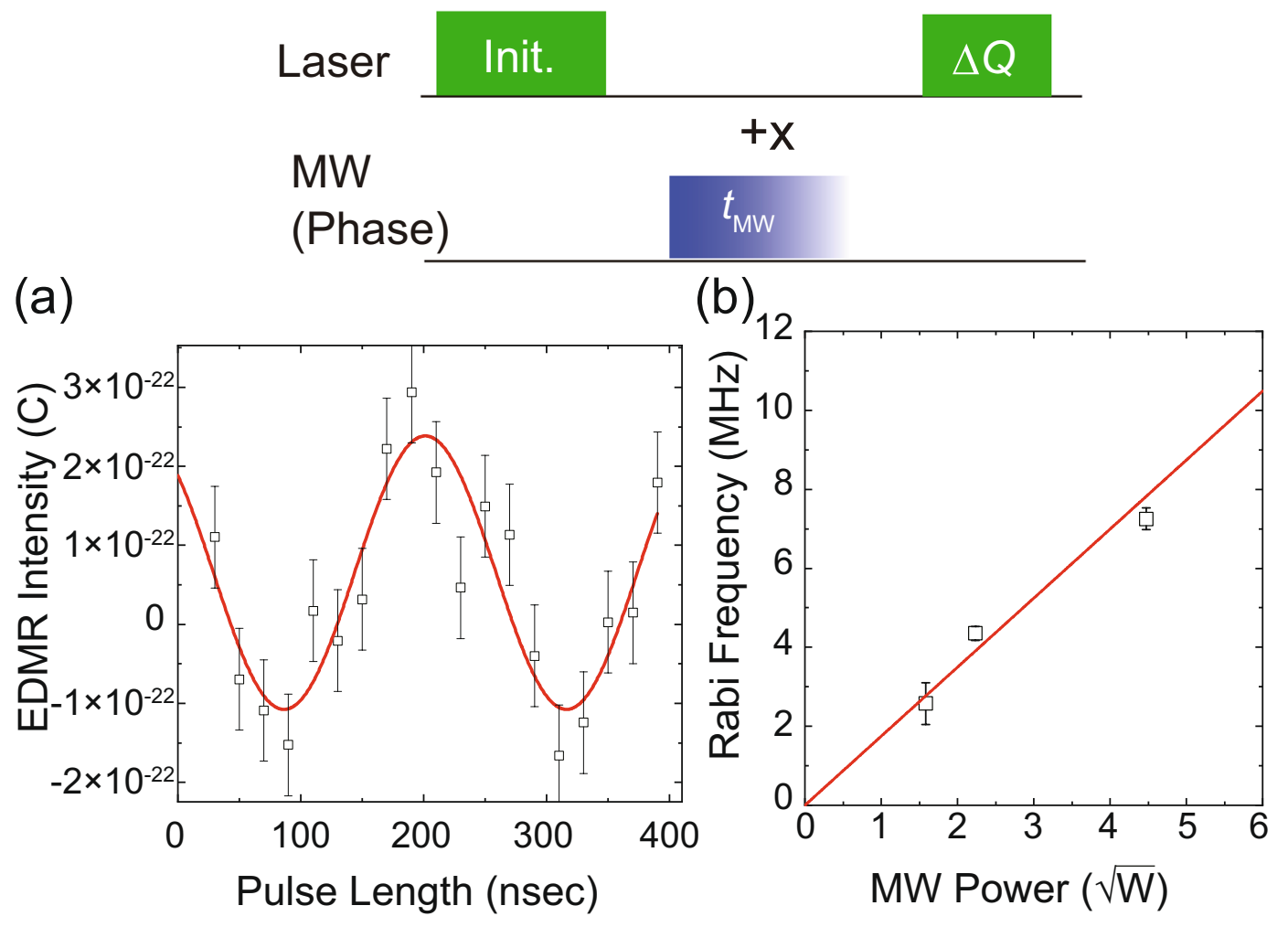

Figure 3. (Top) Pulse sequence of electron-spin Rabi oscillation measurements. $+x$ indicates the phase of MW pulse. (a) Electrically detected electron-spin Rabi oscillation. (b) Rabi frequencies as a function of the square root of MW power.

Fig. 2(b) with the same conditions as the pEDMR measurements. The pEDMR spectrum has different linewidths than the pODMR spectrum. This may be due to the input laser power. While the pEDMR measurements require the illumination of the $30-\mathrm{mW}$ laser to generate the photocurrent, the illumination of the $30-\mathrm{mW}$ laser is strong enough to make broad linewidths in the pODMR signals. However, both spectra have the same resonant frequencies, and hence the NV electron spin resonance signals are observed via the electrical technique.

Rabi oscillations of NV electron spins. The top of Fig. 3 shows the pulse sequence for the electron-spin Rabi oscillations. After the initialisation of the NV electron spins by the first laser pulse, we measured $\Delta Q$ under the application of the last laser as a function of the length of the resonant MW pulse $\left(t_{\mathrm{MW}}\right)$. Here, we set the MW frequency to $2916 \mathrm{MHz}$, corresponding to the transition between $|0\rangle$ and $|+1\rangle$, and its input power to $5 \mathrm{~W}$. The result is shown at the bottom of Fig. 3(a). The solid line in Fig. 3(a) shows the curve fitting result with a sinusoidal curve. We observed an oscillation frequency of $\sim 4.4 \mathrm{MHz}$. Next, we measured the oscillation frequencies with three different input MW powers, as depicted in Fig. 3(b). The figure plots the oscillation frequencies as a function of the square root of the input MW power. The observed data can be fitted by a linear function fixing the intercept at zero, and hence we observed Rabi oscillations of NV electron spins and the Rabi frequencies could be controlled by changing the input MW power.

EDENDOR spectrum of ${ }^{14} \mathrm{~N}$ nuclear spins. To measure the nuclear magnetic resonance of ${ }^{14} \mathrm{~N}$ nuclear spins, we used the ENDOR technique (The detail of the ENDOR is explained in Supplementary Information). This enables the detection of a nuclear magnetic resonance (NMR) signal via a change in the ESE intensity based on the polarisation transfer between the electron and nuclear transitions using the pulse sequence depicted in the top of Fig. $4^{24}$. First, an MW $\pi$-pulse is applied to the transition between $|0\rangle$ to $|+1\rangle$ after illumination by a pulsed laser. This $\pi$-pulse can generate hyperfine coupling between NV electron spins and ${ }^{14} \mathrm{~N}$ nuclear spins and the polarisation between $|+1,0\rangle$ and $|+1,+1\rangle$, where $\left|m_{s}, m_{I}\right\rangle$ are electron and nuclear spins, respectively ${ }^{29-31}$. Then, the RF pulse is applied. Finally, $\Delta Q$ was measured by applying a Hahn echo sequence and the following laser pulse. We observed EDENDOR spectra by measuring $\Delta Q$ as a function of the irradiated RF frequency.

Setting the MW frequency to $2916 \mathrm{MHz}$, the input MW power to $5 \mathrm{~W}$, and the input RF power to $5 \mathrm{~W}$, we observed the spectrum shown in Fig. 4. The observed data can be fitted with the Gaussian function shown by the solid red line in Fig. 4. The curve fitting result shows that we observed a resonance frequency of $3.5 \mathrm{MHz}$. Using equation (S1) in the Supplementary Information, we can estimate that the observed resonance frequency corresponds to the transition between $|+1,0\rangle$ and $|+1,+1\rangle$. Thus, we focus on the transition with the resonance frequency of $3.5 \mathrm{MHz}$ between $|+1,0\rangle$ and $|+1,+1\rangle$ in the following measurements of the Rabi oscillations and the coherence time of the ${ }^{14} \mathrm{~N}$ nuclear spins in this study. 


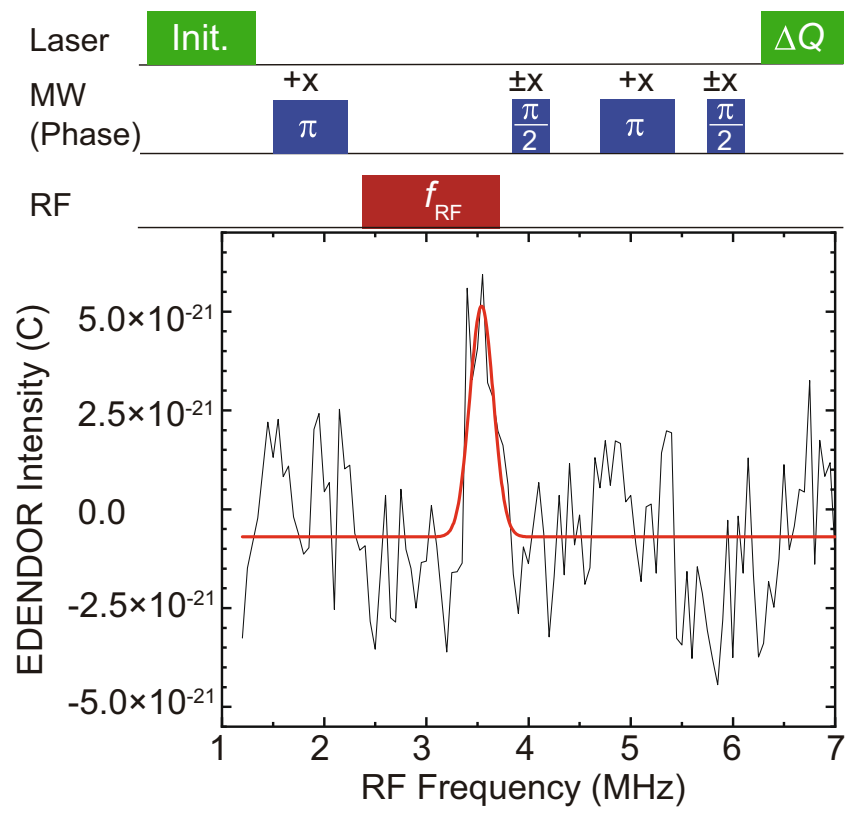

Figure 4. Pulse sequence (top) and the result (bottom) of an electrically detected ENDOR spectrum. $\pm x$ indicate the phase of MW pulse.

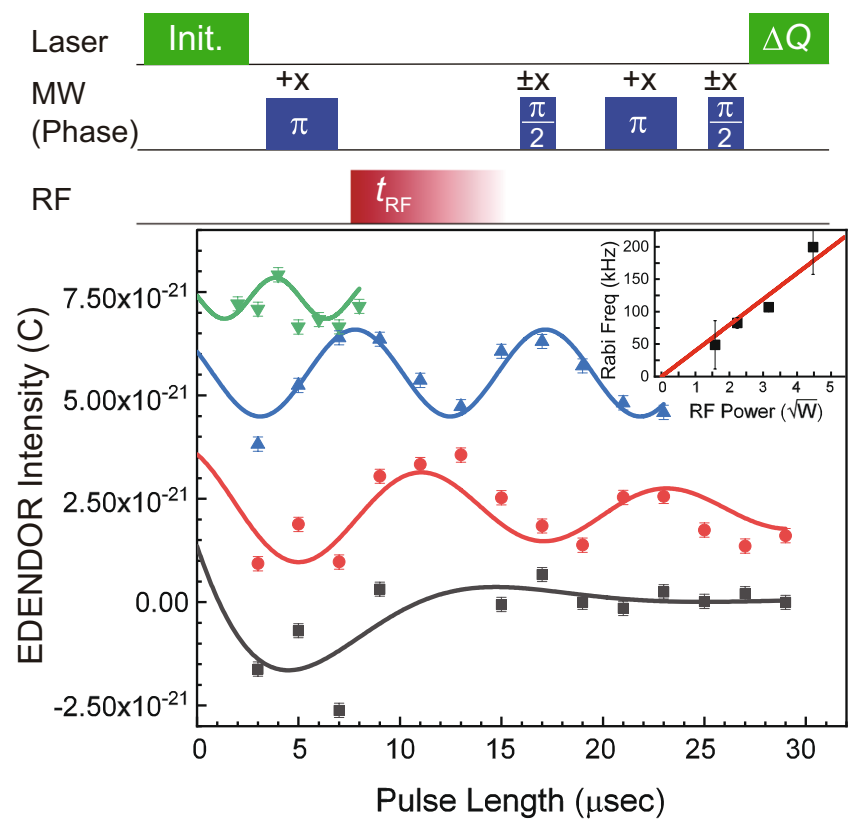

Figure 5. Pulse sequence (top) and the result (bottom) of an electrically detected nuclear Rabi oscillation. $\pm x$ indicate the phase of MW pulse. (Inset) Rabi frequencies as a function of the square root of RF power.

Rabi oscillations of ${ }^{14} \mathrm{~N}$ nuclear spins. The top of Fig. 5 shows the pulse sequence for the measurements of the ${ }^{14} \mathrm{~N}$ nuclear-spin Rabi oscillations. The sequence shows that we measured $\Delta Q$ as a function of the length of the RF pulse. Here, we set the MW frequency to $2916 \mathrm{MHz}$, the input MW power to $5 \mathrm{~W}$, and the RF frequency to $3.5 \mathrm{MHz}$. The results of the electrically detected nuclear-spin Rabi measurements with four different input RF powers are shown in the bottom of Fig. 5 . The red, black, blue, and green points correspond to the results with input $\mathrm{RF}$ powers of $\sim 2.5,5,10$, and $20 \mathrm{~W}$, respectively. The observed oscillations are fitted by sinusoidal curves, which are shown as solid lines in the bottom of Fig. 5. The results of the curve fittings showed that all Rabi oscillations have the same phase offset which depends on the polarisation of ${ }^{14} \mathrm{~N}$ nuclear spin after applying the MW $\pi$ pulse (The detail of the pulse sequence of ENDOR is explained in Supplementary Information). Furthermore, the oscillation frequencies observed by the curve fittings are plotted as a function of the square root of the input RF power in the inset of Fig. 5 . The plots are fitted well by a linear function with the intercept at zero, as shown by the 
(a)
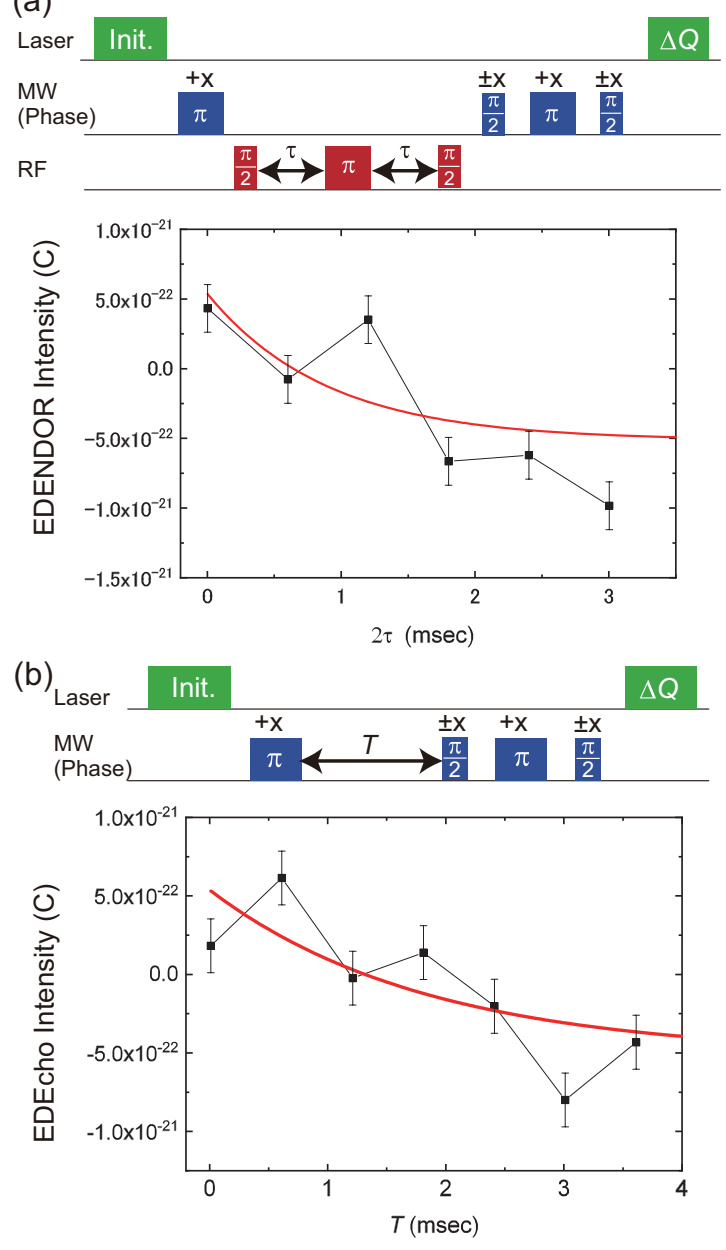

Figure 6. (a) Pulse sequence (top) and the result (bottom) of an electrically detected $T_{2}^{(n)}$ measurement of the ${ }^{14} \mathrm{~N}$ nuclear spins in NV centres. $\pm x$ indicate the phase of MW pulse. (b) Pulse sequence (top) and result and electrically detected $T_{1}^{(e)}$ measurement of the NV electron spins (bottom). $\pm x$ indicate the phase of MW pulse.

solid line, which certifies that the observed oscillations correspond to Rabi oscillations between $|+1,0\rangle$ and $\mid+1$, $+1\rangle$. Hence, the Rabi oscillations of the ${ }^{14} \mathrm{~N}$ nuclear spins can be observed with EDENDOR at room temperature.

Echo decay of ${ }^{14} \mathrm{~N}$ nuclear spins. The top of Fig. 6(a) shows the pulse sequence for a ${ }^{14} \mathrm{~N}$ nuclear $\operatorname{spin}\left(T_{2}^{(n)}\right)$ measurement. It can also be modified based on the EDENDOR sequence to measure $T_{2}^{(n)} 25,26,32$. A nuclear-spin Hahn echo sequence was added between the first MW $\pi$ - and second MW $\pi / 2$-pulse, allowing the nuclear spin echo intensity to be measured via the change in the ESE intensity. We set the input RF power to $10 \mathrm{~W}$ and the other experimental conditions were the same as those for electrically detected nuclear spin Rabi oscillations. Then, we measured $\Delta Q$ as a change of the ESE intensity as a function of the freely evolving time of $2 \tau$. The bottom of Fig. 6(a) shows the result of the $T_{2}^{(n)}$ measurement. The experimentally observed plots are fitted with an exponential curve described by the solid line in Fig. 6(a) fixing the echo amplitude at half the Rabi amplitude. Consequently, $T_{2}^{(n)} \approx 0.9$ (5) ms. Hence, we successfully observed $T_{2}^{(n)}$ with the EDENDOR technique at room temperature.

\section{Discussion}

We here discuss the results of the $T_{2}^{(\mathrm{n})}$ measurement with the modified EDENDOR sequence. After the application of the first MW $\pi$-pulse in the sequence of the $T_{2}^{(\mathrm{n})}$ measurement depicted in Fig. 6(a), the NV electron spins relax back to their thermal equilibrium with a time constant defined as the longitudinal relaxation time $T_{1}^{(\mathrm{e})}$. Thus, the observed $T_{2}^{(n)}$ may be limited by $T_{1}^{(\mathrm{e})}$ in our experiments. We measured $T_{1}^{(\mathrm{e})}$ with the EDMR technique. The pulse sequence for a $T_{1}^{(\mathrm{e})}$ measurement is shown in the top of Fig. 6(b). It is the same as the sequence for $T_{2}^{(n)}$ measurements, without the train of RF pulses. Using the same experimental conditions as those for the $T_{2}^{(n)}$ measurement, except for the irradiation by the RF pulses, we measured $\Delta Q$ as the change in the ESE intensity as a function of $T$, which is the interval between the first MW $\pi$ - and second MW $\pi / 2$-pulses. In the bottom of Fig. 6(b), the experimentally observed data points were fitted with the exponential curve described by the solid line. This gave $T_{1}^{(e)} \approx 1.8(6) \mathrm{ms}$, which is slightly shorter than the reported $T_{1}^{(\mathrm{e}) 12}$, which may be due to the additional decay rate 


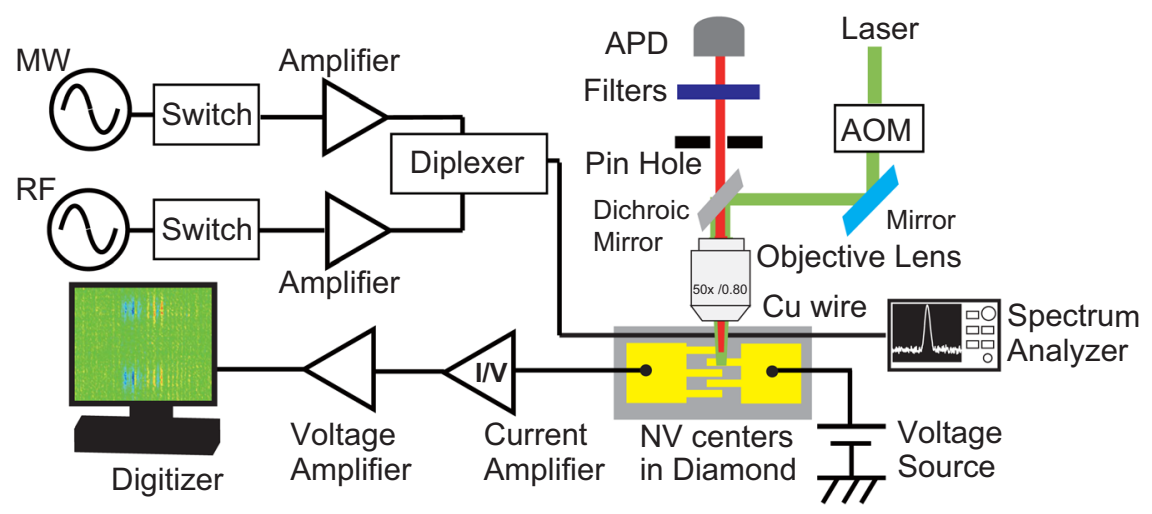

Figure 7. Schematic of the self-built EDENDOR spectrometer.

into $|0\rangle$ due to laser leakage through an acousto-optic modulator (AOM) and/or noises in the magnetic and electric fields from P-donors ${ }^{33,34}$ and/or from a dark electrical current in the absence of the laser illumination. Myers et al. ${ }^{33}$ showed that the electrical field from the diamond surface noise influences the $T_{1}^{(\mathrm{e})}$ of the NV electron spins. Since the electron carriers released from the P-donors and the positive charge of the P-donors after the release generates the electric field, the $T_{1}^{(\mathrm{e})}$ of the NV electron spins may become short in the highly P-doped diamond layer. Moreover, the $T_{1}^{(\mathrm{e})}$ measurement was performed in the presence of a dark current (The details of a dark current are explained in Supplementary Information). The dark current produces magnetic fluctuations ${ }^{34}$, which may shorten $T_{1}^{(\mathrm{e})}$.

Finally, we compare the observed $T_{1}^{(\mathrm{e})}$ with $T_{2}^{(n)}$. When the $T_{2}^{(n)}$ decay is affected only by $T_{1}^{(\mathrm{e})}$, the theory expects $T_{2}^{(n)}=\frac{3}{2} T_{1}^{(e) 10}$. However, the observed $T_{2}^{(n)}$ is slightly shorter than $T_{1}^{(\mathrm{e})}$. When we consider the influence of laser leakage through the AOM accompanied by additional decay rate into $|0\rangle, T_{2}^{(n)}$ might become shorter than $\frac{3}{2} T_{1}^{(e)}$, as discussed in ref. ${ }^{10}$. Thus, precise control of the concentration of P-donors and the upgrade of the EDENDOR spectrometer are important in order to observe long $T_{1}^{(\mathrm{e})}$ and $T_{2}^{(n)}$ by the EDENDOR technique.

In summary, we have demonstrated electrical detection of the Rabi oscillations and $T_{2}^{(n)}$ of the ${ }^{14} \mathrm{~N}$ nuclear spin coherence of an ensemble of NV centres in diamond at room temperature. Using a self-built EDENDOR spectrometer, we observed a signal at $\sim 3.5 \mathrm{MHz}$, which is the ENDOR frequency of the ${ }^{14} \mathrm{~N}$ nuclear spins. This frequency was used to demonstrate electrically detected nuclear-spin Rabi oscillations and $T_{2}^{(n)}$ measurements of the ${ }^{14} \mathrm{~N}$ nuclear spins at room temperature. We observed $T_{2}^{(n)} \approx 0.9 \mathrm{~ms}$. This study should contribute to the development of future electron- and nuclear-spin based diamond quantum devices.

\section{Method}

Sample preparation. In this study, we used an ensemble of NV centres in a P-doped $n$-type diamond layer. This sample has two characteristics: (1) a negative charge state of the NV centre $\left(\mathrm{NV}^{-}\right)$in the P-doped $n$-type diamond that is stable even under 532-nm laser illumination ${ }^{35}$ and (2) the highly P-doped $n$-type diamond has high electrical conductivity ${ }^{36}$. The ensemble of NV centres in the $n$-type diamond was prepared via the following processes. A P-doped $n$-type diamond layer (10- $\mu$ m thick) was synthesised on a type IIa (001) diamond substrate by chemical vapour deposition (CVD) ${ }^{36}$. The $n$-type diamond layer has a P-donor concentration of $\sim 10^{18} \mathrm{~cm}^{-3}$. The ensemble of NV centres was made by ${ }^{14} \mathrm{~N}^{+}$-ion implantation (dose: $1 \times 10^{15} \mathrm{~cm}^{-2}$ ) with a kinetic energy of $350 \mathrm{keV}$, followed by annealing at $1000^{\circ} \mathrm{C}$ for 1 hour under vacuum. We estimated the concentration of NV centres in the detection volume of the self-built EDENDOR spectrometer. The detection volume of the confocal microscope used as a laser illumination unit in the EDENDOR spectrometer can be estimated as $2 \times 10^{-12} \mathrm{~cm}^{3}$ (Details are explained in Supplementary Information). When the fluorescence from the NV centre is proportional to the concentration of NV centres, we can estimate the concentration of NV centres of $1 \times 10^{15} \mathrm{~cm}^{-3}\left(2 \times 10^{3}\right.$ $\mathrm{NV}$ centres in the spot size of the focused laser beam). After generating the ensemble of NV centres, interdigital contacts with $\sim 2-\mu \mathrm{m}$ gaps were fabricated on the $n$-type diamond layer to detect photocurrent changes, by the following three steps: (1) electron-beam lithography, (2) deposition of $\mathrm{Ti}(30 \mathrm{~nm}) / \mathrm{Pt}(30 \mathrm{~nm}) / \mathrm{Au}(100 \mathrm{~nm})$ multilayers, and (3) annealing at $420^{\circ} \mathrm{C}$ for $30 \mathrm{~min}$ under an argon atmosphere. Details of the electrical characteristics of the electrical contacts are given in Supplementary Information.

Self-built EDENDOR spectrometer. Figure 7 shows a self-built EDENDOR spectrometer (Details are explained in Supplementary Information). This spectrometer consists of (1) a laser illumination unit of a confocal microscope with a 532-nm laser to generate a photocurrent from an ensemble of NV centres, (2) a microwave (MW) and radio-frequency (RF) irradiation unit to manipulate the NV electron and ${ }^{14} \mathrm{~N}$ nuclear spins, and (3) a photocurrent detection unit. Using the laser illumination unit, the laser illuminates the ensemble of NV centres, focused by an objective lens with an NA of 0.8 . Then, a photocurrent is generated from the NV centres. The NV centres also emit photons under the laser illumination, which are detected by an avalanche photodiode (APD) after passing through a pinhole and a filter (633-nm long-pass filter). In this study, the APD is used to fix the position of the illumination spot in a place between the electrical contacts by monitoring the photons from the $\mathrm{NV}$ centres. MW and RF are combined with a frequency diplexer and irradiated to the NV centres with a $\sim 50-\mu \mathrm{m}$ 
copper wire. The irradiated MW and RF frequencies and powers are measured by a spectrum analyser during the EDMR and EDENDOR measurements. The change of photocurrent is measured under the application of a constant voltage of $8 \mathrm{~V}$. The change of photocurrent is converted into a change of voltage by a current amplifier. Then, it can be measured by a digitiser on a personal computer after amplification by a voltage amplifier. In this study, we measured $\sim 2 \times 10^{3} \mathrm{NV}$ centres in the EDMR and EDENDOR measurements and observed their signals from $10^{7}$ or more repetitions. A phase cycling technique is applied to subtract the artefact noises due to on- and off-resonant MW and RF contributions and laser-power fluctuations from the EDMR and EDENDOR signals ${ }^{27,32,37}$ (The details of the phase cycling are explained in Supplementary Information). Note that the phases of the MW pulses are indicated by $\pm x$ on the MW pulses shown in Figs. 2-6.

\section{Data availability}

The data that support the findings of this study are available from the corresponding author upon reasonable request.

Received: 3 September 2019; Accepted: 6 January 2020;

Published online: 21 January 2020

\section{References}

1. Yusa, G., Muraki, K., Takashina, K., Hashimoto, K. \& Hirayama, Y. Controlled multiple quantum coherences of nuclear spins in a nanometre-scale device. Nature 434, 1001 (2005).

2. Morton, J. J. L. et al. Solid-state quantum memory using the ${ }^{31} \mathrm{P}$ nuclear spin. Nature 455, 1085 (2008).

3. Fuchs, G. D., Burkard, G., Klimov, P. V. \& Awschalom, D. D. A quantum memory intrinsic to single nitrogen-vacancy centres in diamond. Nat. Phys. 7, 789 (2011)

4. Maurer, P. C. et al. Room-temperature quantum bit memory exceeding one second. Science 336, 1283-6 (2012)

5. Pla, J. J. et al. High-fidelity readout and control of a nuclear spin qubit in silicon. Nature 496, 334 (2013).

6. Saeedi, K. et al. Room-Temperature Quantum Bit Storage Exceeding 39 Minutes Using Ionized Donors in Silicon-28. Science 342, 830 (2013).

7. Sigillito, A. J., Tyryshkin, A. M., Schenkel, T., Houck, A. A. \& Lyon, S. A. All-electric control of donor nuclear spin qubits in silicon. Nat. Nanotech. 12, 958 (2017).

8. Zaiser, S. et al. Enhancing quantum sensing sensitivity by a quantum memory. Nat. Commun. 7, 12279 (2016).

9. Matsuzaki, Y. et al. Hybrid quantum magnetic field sensor with an electron spin and a nuclear spin in diamond. Phys. Rev. A 94, 052330 (2016).

10. Pfender, M. et al. Nonvolatile nuclear spin memory enables sensor-unlimited nanoscale spectroscopy of small spin clusters. Nat. Commun. 8, 834 (2017).

11. Yang, S. et al. High-fidelity transfer and storage of photon states in a single nuclear spin. Nat. Photon. 10, 507 (2016).

12. Neumann, P. et al. Multipartite entanglement among single spins in diamond. Science 320, 1326-9 (2008).

13. Waldherr, G. et al. Quantum error correction in a solid-state hybrid spin register. Nature 506, 204 (2014).

14. Balasubramanian, G. et al. Ultralong spin coherence time in isotopically engineered diamond. Nat. Mater. 8, 383-7 (2009).

15. Herbschleb, E. D. et al. Ultra-long coherence times amongst room-temperature solid-state spins. Nat. Commun. 10, 3766 (2019).

16. Mizuochi, N. et al. Coherence of single spins coupled to a nuclear spin bath of varying density. Phys. Rev. B 80, 041201(R) (2009).

17. Doherty, M. W. et al. The nitrogen-vacancy colour centre in diamond. Phys. Rep. 528, 1 (2013).

18. Bourgeois, E. et al. Photoelectric detection of electron spin resonance of nitrogen-vacancy centres in diamond. Nat. Commun. $\mathbf{6}$, 8577 (2015).

19. Hrubesch, F. M., Braunbeck, G., Stutzmann, M., Reinhard, F. \& Brandt, M. S. Efficient Electrical Spin Readout of NV- Centers in Diamond. Phys. Rev. Lett. 118, 037601 (2017).

20. Gulka, M. et al. Pulsed Photoelectric Coherent Manipulation and Detection of N- V Center Spins in Diamond. Phys. Rev. Applied 7, 044032 (2017).

21. Siyushev, P. et al. Photoelectrical imaging and coherent spin-state readout of single nitrogen-vacancy centers in diamond. Science 363, 728 (2019).

22. Stich, B., Greulich-Weber, S. \& Speath, J.-M. Electrical detection of electron nuclear double resonance in silicon. Appl. Phys. Lett. 68, 1102 (1996).

23. McCamey, D. R., van Tol, J., Morley, G. W. \& Boehme, C. Electronic Spin Storage in an Electrically Readable Nuclear Spin Memory with a Lifetime> 100 Seconds. Science 330, 1652 (2010).

24. Hoehne, F., Dreher, L., Huebl, H., Stutzmann, M. \& Brandt, M. S. Electrical Detection of Coherent Nuclear Spin Oscillations in Phosphorus-Doped Silicon using Pulsed ENDOR. Phys. Rev. Lett. 106, 187601 (2011)

25. McCamey, D. R., Boehme, C., Morley, G. W. \& van Tol, J. Electrically detected spin echoes of donor nuclei in silicon. Phys. Rev. B 85, 073201 (2012).

26. Dreher, L., Hoehne, F., Stutzmann, M. \& Brandt, M. S. Nuclear Spins of Ionized Phosphorus Donors in Silicon. Phys. Rev. Lett. 108, 027602 (2012).

27. Malissa, H. et al. Room-temperature coupling between electrical current and nuclear spins in OLEDs. Science 345, 1487 (2014).

28. Boehme, C. \& Lips, K. Theory of time-domain measurement of spin-dependent recombination with pulsed electrically detected magnetic resonance. Phys. Rev. B 68, 245105 (2003).

29. He, X.-F., Manson, N. B. \& Fisk, P. T. H. Paramagnetic resonance of photoexcited N-V defects in diamond. II. Hyperfine interaction with the $14 \mathrm{~N}$ nucleus. Phys. Rev. B 47, 8816 (1993).

30. Felton, S. et al. Hyperfine interaction in the ground state of the negatively charged nitrogen vacancy center in diamond. Phys. Rev. B 79, 075203 (2009).

31. Yavkin, B. V., Mamin, G. V. \& Orlinskii, S. B. High-frequency pulsed ENDOR spectroscopy of the NV- centre in the commercial HPHT diamond. J. Magn. Reson. 262, 15 (2016).

32. Hoehne, F. et al. Time constants of spin-dependent recombination processes. Phys. Rev. B 88, 155301 (2013).

33. Myers, B. A., Ariyaratne, A. \& Jayich, A. C. B. Double-Quantum Spin-Relaxation Limits to Coherence of Near-Surface NitrogenVacancy Centers. Phys. Rev. Lett. 118, 197201 (2017).

34. Ariyaratne, A., Bluvstein, D., Myers, B. A. \& Jayich, A. C. B. Nanoscale electrical conductivity imaging using a nitrogen-vacancy center in diamond. Nat. Commun. 10, 1038 (2018).

35. Doi, Y. et al. Pure negatively charged state of the NV center in $n$-type diamond. Phys. Rev. B 93, 081203(R) (2016).

36. Kato, H., Yamasaki, S. \& Okushi, H. $n$-type doping of (001) oriented single-crystalline diamond by phosphorus. Appl. Phys. Lett. 86, 222111 (2005).

37. Schweiger, A. \& Jeschke, G. Principles of pulse electron paramagnetic resonance (Oxford University Press, New York, 2001). 


\section{Acknowledgements}

We wish to acknowledge T. Ono and T. Moriyama for their support with the fabrication and evaluation of the electrical contacts. This work was supported by CREST (No. JPMJCR1333), KAKENHI (No. 15H05868), MEXT Q-LEAP (No. JPMXS0118067395), Kyoto University Nano Technology Hub, and the Collaborative Research Program of the Institute for Chemical Research, Kyoto University (Grant No. 2017-78). HM acknowledges support from KAKENHI (No. 16K17848 and No. 19H02546), Murata Science Foundation, and Iketani Science and Technology Foundation.

\section{Author contributions}

H.M., performed the fabrication of electrical contacts, measurements, and the data analysis assisted by S.K. and M.F. and N.M.; H.K. grew the phosphorus-doped diamond, assisted by T.M. and S.Y.; H.M. and N.M. wrote the manuscript, and all the authors (H.M., S.K., M.F., H.K., T.M., S.Y. and N.M.) discussed it.

\section{Competing interests}

The authors declare no competing interests.

\section{Additional information}

Supplementary information is available for this paper at https://doi.org/10.1038/s41598-020-57569-8.

Correspondence and requests for materials should be addressed to H.M. or N.M.

Reprints and permissions information is available at www.nature.com/reprints.

Publisher's note Springer Nature remains neutral with regard to jurisdictional claims in published maps and institutional affiliations.

Open Access This article is licensed under a Creative Commons Attribution 4.0 International License, which permits use, sharing, adaptation, distribution and reproduction in any medium or format, as long as you give appropriate credit to the original author(s) and the source, provide a link to the Creative Commons license, and indicate if changes were made. The images or other third party material in this article are included in the article's Creative Commons license, unless indicated otherwise in a credit line to the material. If material is not included in the article's Creative Commons license and your intended use is not permitted by statutory regulation or exceeds the permitted use, you will need to obtain permission directly from the copyright holder. To view a copy of this license, visit http://creativecommons.org/licenses/by/4.0/.

(C) The Author(s) 2020 